\title{
Geomorfología y forma urbana. Comportamiento térmico de distintas tramas en áreas de piedemonte: el caso de Mendoza, Argentina
}

Ana Castillo. Instituto de Ambiente, Hábitat y Energía (INAHE), Mendoza, Argentina. Érica Correa. Instituto de Ambiente, Hábitat y Energía (INAHE), Mendoza, Argentina. María Cantón. Instituto de Ambiente, Hábitat y Energía (INAHE), Mendoza, Argentina.

RESUMEN | El crecimiento urbano sobre el piedemonte del área metropolitana de Mendoza, Argentina, ha modificado las condiciones del territorio y afectado el balance energético en él, dando como resultado un incremento de la temperatura. Esto produce un mayor consumo de energía para el acondicionamiento térmico de verano y empeora la habitabilidad térmica de los espacios públicos. Para detectar configuraciones urbanas que permitan mitigar este impacto, se analizó la interacción entre los parámetros microclimáticos y variables geomorfológicas que definen el comportamiento térmico de los espacios urbanos. Se seleccionaron, caracterizaron geomorfológica, morfológica y tecnológicamente, y monitorearon microclimáticamente, ocho canales viales urbanos representativos insertos en diferentes tipologías de tramas, presentes en el área de estudio. Los datos fueron evaluados estadísticamente mediante análisis multivariados. La investigación pone en relevancia el impacto de las decisiones de diseńo al momento de priorizar estrategias para alcanzar la sustentabilidad del ambiente construido en áreas residenciales localizadas en el piedemonte.

PALABRAS CLAVE | morfología urbana, sustentabilidad urbana, medioambiente urbano.

ABSTRACT | The urban growth that takes place on the piedmont of the metropolitan area of Mendoza, Argentina has modified the conditions of the territory and the energy balance, resulting in an increase of temperature. This produces an increased energy consumption for summer thermal conditioning and deteriorates the thermal habitability of public spaces. To detect urban configurations that mitigate this impact, the interaction between microclimatic parameters and geomorphological variables that define the thermal behavior of urban spaces were analyzed. Eight representative urban canyons inserted in different types of frames, present in the study area, were selected, characterized geomorphologically, morphologically and technologically, and monitored microclimatically. The data were evaluated statistically by multivariate analysis. The research highlights the impact of design decisions when prioritizing strategies to achieve the sustainability of the built environment in residential areas located in the piedmont.

KEYWORDS | urban morphology, urban sustainability, urban environment

Recibido el 14 de noviembre de 2017, aprobado el 10 de julio de 2018.

E-mails: A. Castillo, alcastillo@mendoza-conicet.gob.ar |É. Correa, ecorrea@mendoza-conicet.gob.ar |

M. Cantón,macanton@mendoza-conicet.gob.ar 


\section{Introducción}

El fenómeno urbano y su planificación constituyen un asunto complejo, sometido a diversas influencias y decisiones vinculadas a variados puntos de vista: sociales, económicos, políticos, administrativos y jurídicos, entre otros. Durante las últimas décadas, las ciudades latinoamericanas han experimentado un marcado proceso de crecimiento como consecuencia directa de la mutación de las estructuras económicas y de la alta tasa demográfica. Hoy, casi ocho de cada diez latinoamericanos vive en ciudades, cifra que se ha incrementado siete veces desde los ańos cincuenta (Libertun de Duren, 2014).

Distintas zonas de América, y de modo particular la ciudad de Mendoza, Argentina, han coincidido en el modelo expansionista de crecimiento urbano. En varios casos tal expansión se da tanto sobre áreas cultivadas como sobre el piedemonte, lo que provoca la degradación de los sistemas naturales próximos a los límites de las ciudades, afectando las condiciones medioambientales de las mismas (Castillo, Correa \& Cantón, 2017; Romero \& Vásquez, 2005). Como resultado se obtiene un bajo nivel de eficiencia en la utilización de los recursos y funciones ambientales que ofrecen los sistemas biofísicos de la ciudad (Welz \& Krellenberg, 2016).

En este sentido, los postulados de la arquitectura y urbanismo sostenibles buscan optimizar las relaciones entre la ciudad y sus sistemas ambientales a fin de lograr el aprovechamiento adecuado de los recursos naturales locales, equilibrando las características del diseńo urbano con las variables climáticas, topográficas y territoriales (López \& López, 2004).

El área metropolitana de Mendoza (АмM), localizada en el centro oeste de Argentina, no ha estado ajena a esta problemática. Su clima es de tipo árido y, geomorfológicamente, se caracteriza por un cordón montańoso al oeste que decrece hacia el este hasta formar mesetas. Concentra el 62,8\% del total de la población de Mendoza y durante la última década su población urbana se incrementó un 9,4\% (Instituto Nacional de Estadísticas y Censos [Indec], 2010).

Los asentamientos del conglomerado urbano del Амм se han expandido en dos direcciones, una hacia el oasis irrigado y otra hacia el oeste, ascendiendo sobre el piedemonte. El 99\% de este territorio responde al desarrollo edilicio en baja densidad (viviendas unifamiliares de uno o dos niveles) (Instituto Provincial de la Vivienda [IPV], 2010).

$\mathrm{Al}$ no contar con una clara normativa en relación con el ordenamiento territorial en el sector de piedemonte, la denominada "Ciudad-Oasis" ha crecido sobre dicho sector, y lo ha hecho siempre a partir de su tradición urbanística de llanura, no advirtiéndose a tiempo que un territorio distinto requiere respuestas distintas (Abraham, Roig \& Salomón, 1990). Entre los impactos y conflictos más importantes del piedemonte mendocino se encuentra la urbanización acelerada y no planificada, lo que se manifiesta tanto en asentamientos espontáneos como en barrios de origen público y privado (figura 1). Todos ellos se caracterizan por procesos de urbanización no adaptados al ambiente pedemontano, con escasos recursos de agua y suelo, destrucción de la vegetación natural, aceleración de la escorrentía y degradación del paisaje (Castillo, Correa \& Cantón, 2016) 
FIGURA I | Crecimiento urbano sobre el área de piedemonte

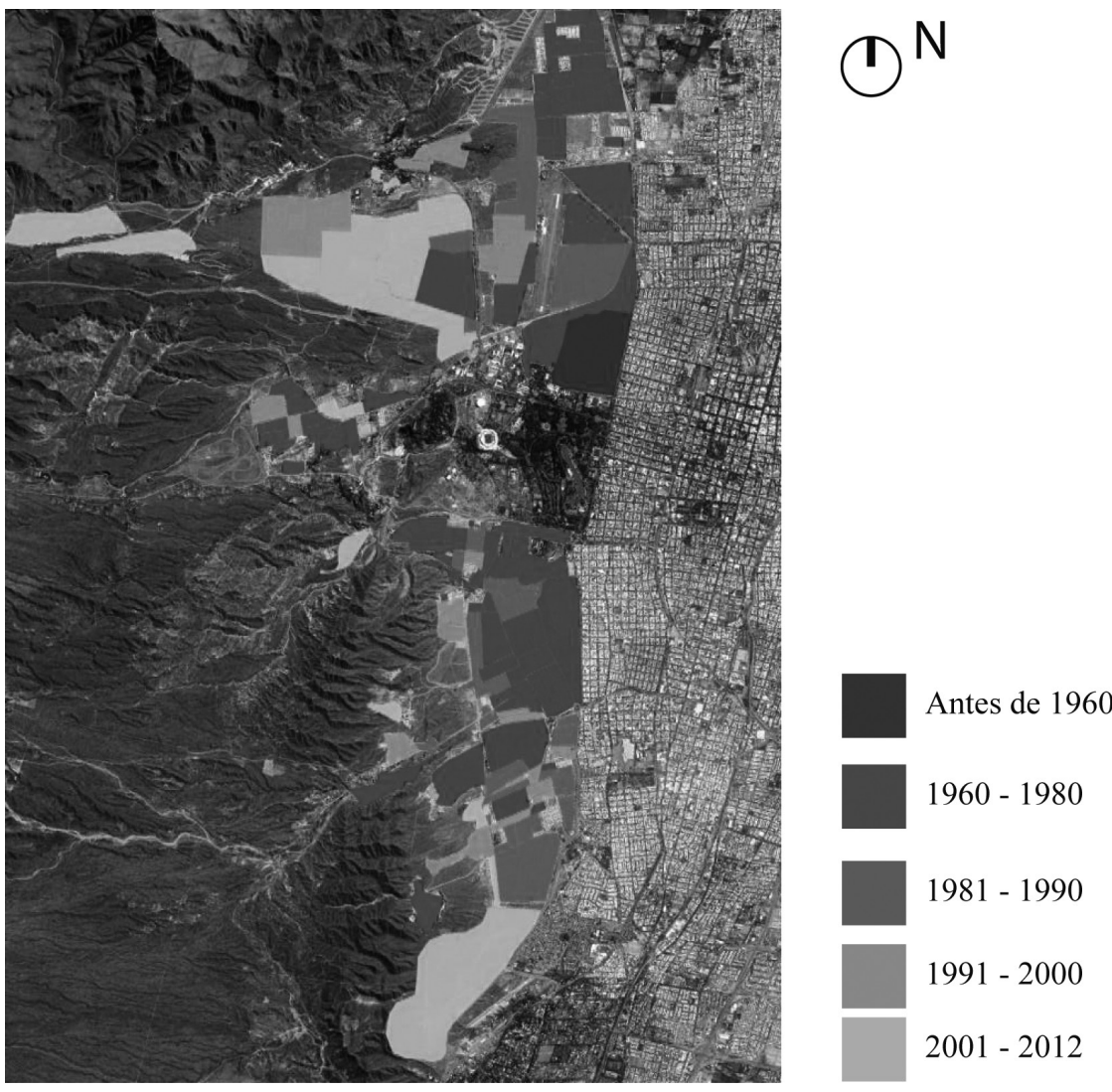

FUENTE ELABORACIÓN PROPIA CON BASE EN SECRETARÍA DE AMBIENTE Y ORDENAMIENTO TERRITORIAL (2OI7), Sistema DE INFORMACión AMBIENTAL TERRITORIAL (SIAT), PIED-CRECIMIENTO URBANO

En este sentido, es imperante intervenir sobre los criterios de diseńo urbano de los conjuntos residenciales que se desarrollen sobre el piedemonte del AMM, a fin de mitigar aspectos negativos como la erosión del terreno, la cual ocasiona deslizamientos de tierra y sellamiento de la misma. Esto propicia las inundaciones, causando desertificación por la modificación del ecosistema, empobrecimiento de los recursos climáticos de la región -por ejemplo, modificación de los perfiles de viento, la calidad del aire y las condiciones de confort- e incremento de los consumos energéticos para el acondicionamiento térmico edilicio, entre otros.

Considerando lo anterior, el presente trabajo tiene por objetivo analizar la interacción entre los parámetros microclimáticos, las variables geomorfológicas y las características de la trama que definen el comportamiento térmico de los espacios urbanos ubicados en el piedemonte. El objetivo final es identificar y proponer esquemas morfológicos y tecnológicos que contribuyan a mejorar la temperatura exterior y el grado de habitabilidad térmica en épocas estivales, que son consideradas como las más demandantes para ciudades de clima árido. 


\section{Metodología}

El trabajo tiene por objeto determinar los factores que influyen en mayor grado sobre el comportamiento térmico de los espacios urbanos de conjuntos residenciales ubicados en el piedemonte de la ciudad de Mendoza.

Metodológicamente, mediante el análisis estadístico, busca establecer la influencia sobre la temperatura del aire que tiene un conjunto de variables y/o indicadores que caracterizan las condiciones microclimáticas, geomorfológicas, morfológicas y tecnológicas de los casos de estudio, lo que influye directamente en la habitabilidad de los espacios exteriores.

El análisis estadístico se realizó a partir de un conjunto de datos obtenidos de una secuencia de actividades, que incluyen la selección de los casos de estudio, la caracterización de los casos y el monitoreo microclimático. La metodología empleada para el desarrollo de las mismas se describe a continuación.

\section{Selección de casos de estudio}

Con el objeto de caracterizar los tipos de tramas existentes en el piedemonte, se realizó un relevamiento de tipologías de manzanas. El término 'piedemonte' es utilizado para indicar el punto donde nace una montańa, o la llanura formada al pie de un macizo montañoso por los conos aluvionales. De acuerdo con la definición de Abraham, Roig y Salomón (1990), el piedemonte mendocino está ubicado en la precordillera de Mendoza y es una estrecha franja de 15 a $20 \mathrm{~km}$ de ancho, que se extiende entre la vertiente oriental de las sierras y la llanura, aproximadamente entre los 1.500 y los 800 m.s.n.m. Constituye un ambiente frágil e inestable debido a sus grandes pendientes, lluvias estivales violentas y concentradas en sectores parciales de la cuenca. Posee vegetación escasa y degradada, sus suelos son no consolidados y factibles de padecer acelerados procesos de erosión, principalmente hídrica. Desde el punto de vista climático prevalece la condición árida, por la escasez de recursos hídricos: no existen en el piedemonte cursos de agua permanentes, solo vertientes de escaso caudal y cauces temporarios, torrentes o wadis (cauces secos o estacionales de ríos, arroyos y torrentes que drenan regiones cálidas y áridas). Las consecuencias de los impactos mencionados se traducen en la acentuación de los riesgos de tipo aluvional y los de tipo microclimático, representados, entre otros, por la magnitud y localización del efecto de la isla de calor. Estudios del fenómeno de la isla de calor urbana para la ciudad de Mendoza revelan el impacto negativo que en los sectores periurbanos tiene la urbanización sobre los perfiles de temperatura de la zona correspondiente al piedemonte, donde la magnitud de la isla de calor puede alcanzar hasta $9^{\circ} \mathrm{C}$. De acuerdo con los fenómenos de transferencia de energía, la intensa magnitud de la isla de calor diurna sobre este sector se explica considerando que el sellamiento de suelo ocasionado por la urbanización desmedida del mismo, sumado a las propiedades térmicas de los materiales que lo componen (ripio, roca y suelos pobres), aumentan la inercia térmica del lugar, que extiende su periodo de enfriamiento hasta las primeras horas de la mañana (Correa, De Rosa \& Lesino, 2006).

El relevamiento se llevó a cabo a partir de la utilización de planos catastrales del амм proporcionados por la Dirección General de Catastro de la Provincia de 
Mendoza. De acuerdo con estos planos, se identificó que la trama más distintiva del desarrollo urbano de este sector es la racional con orientaciones variadas ( $\mathrm{N}-\mathrm{S}$, E-O, NE-SO y NO-SE). A partir de esta información se escogieron cuatro conjuntos urbanos, los cuales representan barrios ya consolidados ubicados en el sector norte del AMM, de origen público y que cuentan con toda la infraestructura, equipamiento urbano y servicios necesarios para su ocupación. La selección de estos conjuntos también tuvo que ver con su altura sobre el nivel del mar. De cada uno de ellos se escogieron dos tipologías diferentes de canales viales urbanos (Cvu), dando un total de ocho casos analizados. Esas tipologías, ambas de trama racional y que fueron elegidas por ser las que predominan en el área de piedemonte, se diferencian por las orientaciones de sus canales (figura 2). Esta selección se debe a que el AMM presenta características de homogeneidad en relación con el sistema constructivo, y también a su densidad (más del 90\% del total del área urbanizada es de baja densidad: uno o dos niveles de altura). Sin embargo, manifiesta una gran heterogeneidad en relación con la forma y orientación de las manzanas, ancho y largo de calles, y distribución de la edilicia respecto de la trama. Estas características dificultan contrastar directamente los casos, lo que implica que, para determinar los mejores esquemas en términos de comportamiento térmico, se requiere de un análisis sistemático de los atributos de los cvu y sus efectos sobre el microclima.

\section{FIGURA 2 | Ubicación e identificación de los casos de estudio}

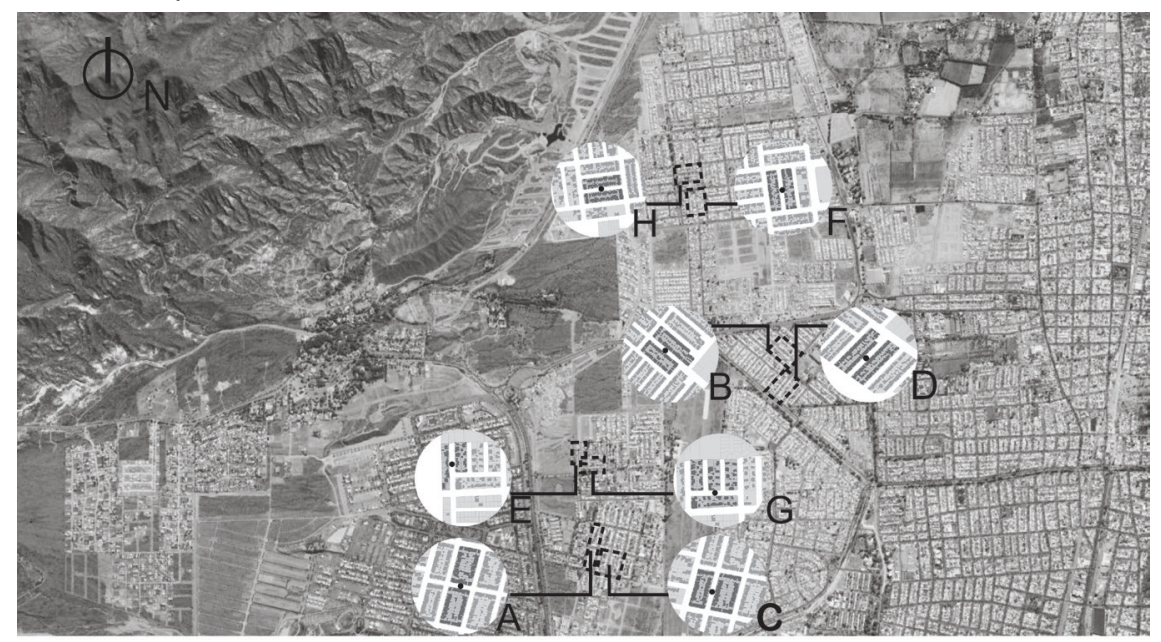

FUENTE ELABORACIÓN PROPIA

\section{Caracterización}

De acuerdo con los objetivos del trabajo, se seleccionaron y caracterizaron ocho canales viales urbanos insertos en una tipología de trama racional, pero con diferentes orientaciones y distribución respecto de la trama y de la edilicia. La caracterización se realizó mediante variables o descriptores acordes con la configuración urbana, el perfil urbano, las características tecnológicas, los sistemas constructivos, 
la distribución dentro de la manzana. Para ello se estableció un conjunto de indicadores geomorfológicos, morfológicos, tecnológicos (materialidad y forestación):

- Geomorfológicos: altitud (Al), establece la altitud sobre el nivel del mar; azimut $(\mathrm{Az})$ es indicativo de la orientación del canal urbano analizado, en relación con el norte geográfico.

- Morfológicos: largo (L), ancho (A), superficie de canal vial urbano (SCVU), relación altura de viviendas con ancho de canal $(\mathrm{H} / \mathrm{W})$, superficie total construida (STC), superficie total de patios (STP), superficie de patios perimetrales (SPP), superficie de patios centrales (SPC), relación entre patios perimetrales y centrales (RPPC), compacidad (C), volumen total construido (VTC), densidad edilicia (DE), factor de ocupación de superficie (FOs), horas sol (HS).

- Tecnológicos: factor de visión de cielo (svf), número de forestales (NoF) y albedo (Alb). Se utilizó el albedo - porcentaje de radiación que una superficie refleja respecto a la radiación que incide sobre ella- como variable para caracterizar el comportamiento tecnológico de los casos; al respecto, la tecnología utilizada (ladrillo, revoque) es semejante en todos los canales analizados. Se considera que el comportamiento radiativo de las superficies (inercia térmica y conductividad) es el factor más importante de los que inciden en el comportamiento térmico de los espacios urbanos, y está determinado tanto por el color como por el acabado de los elementos constructivos.

Es importante señalar cómo se calcula cada uno de los indicadores: la superficie del cvu es la sumatoria de las superficies de las parcelas más las aceras y calzada; la compacidad es la división entre el volumen total construido dividido por la superficie del CVu; el volumen total construido (VTC) es la resultante entre la superficie construida multiplicada por la altura de la edilicia; la densidad edilicia es la fracción entre el número total de viviendas dividido por la superficie del canal. El indicador SVF es el factor que refleja la visión de bóveda celeste de los cańones urbanos, y fue calculado a partir de imágenes hemisféricas capturadas con una cámara digital NikonRCoolPix, equipada con un lente ojo de pez, y procesadas con el software Pixel de Cielo desarrollado por Correa, Pattini y Fornés (2006). Este software obtiene el valor de SVF en condiciones de cielo despejado, con forestación urbana intensa y en ciudades con alta reflectividad, típica de regiones áridas, como es el caso de esta área de estudio. 


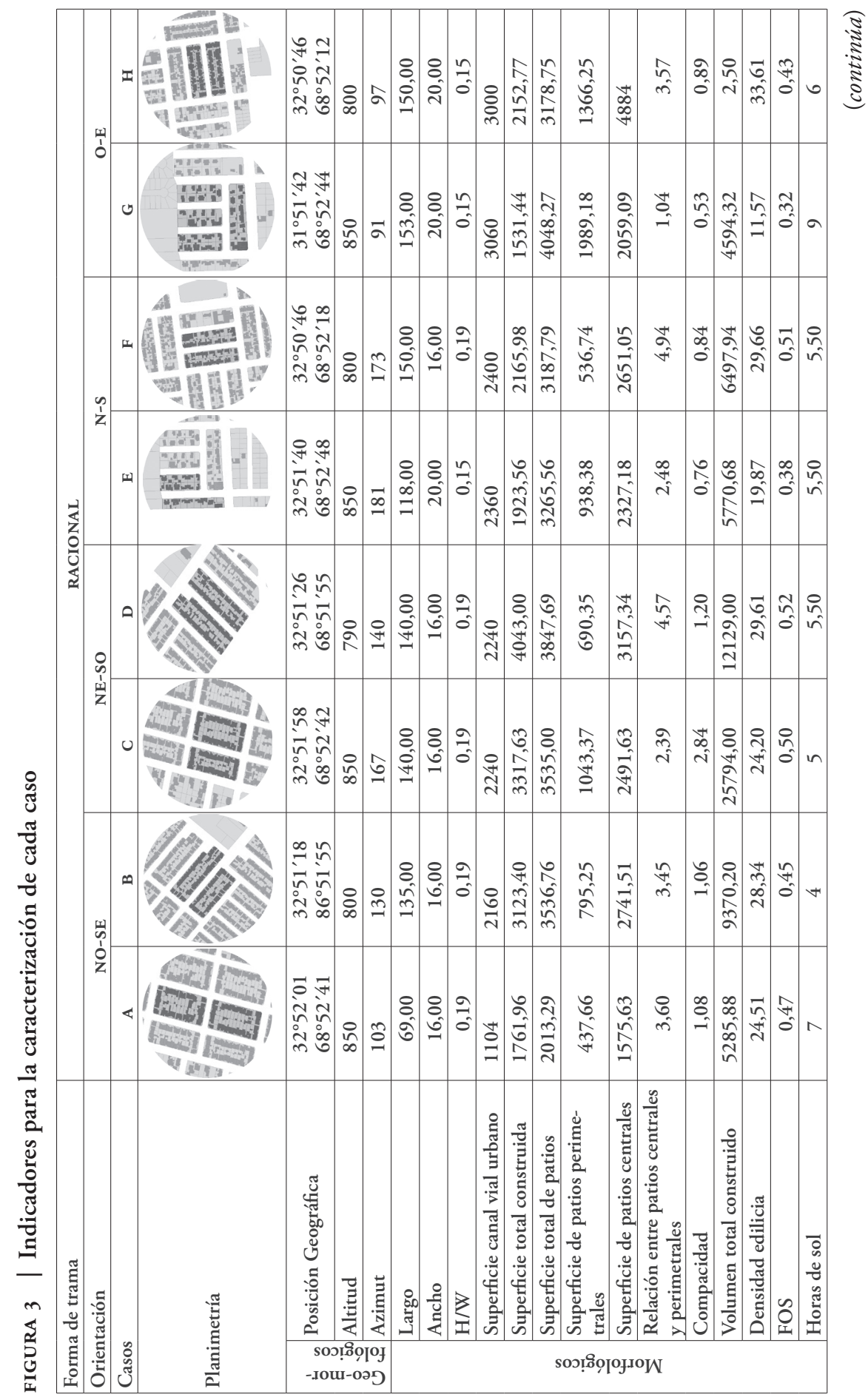




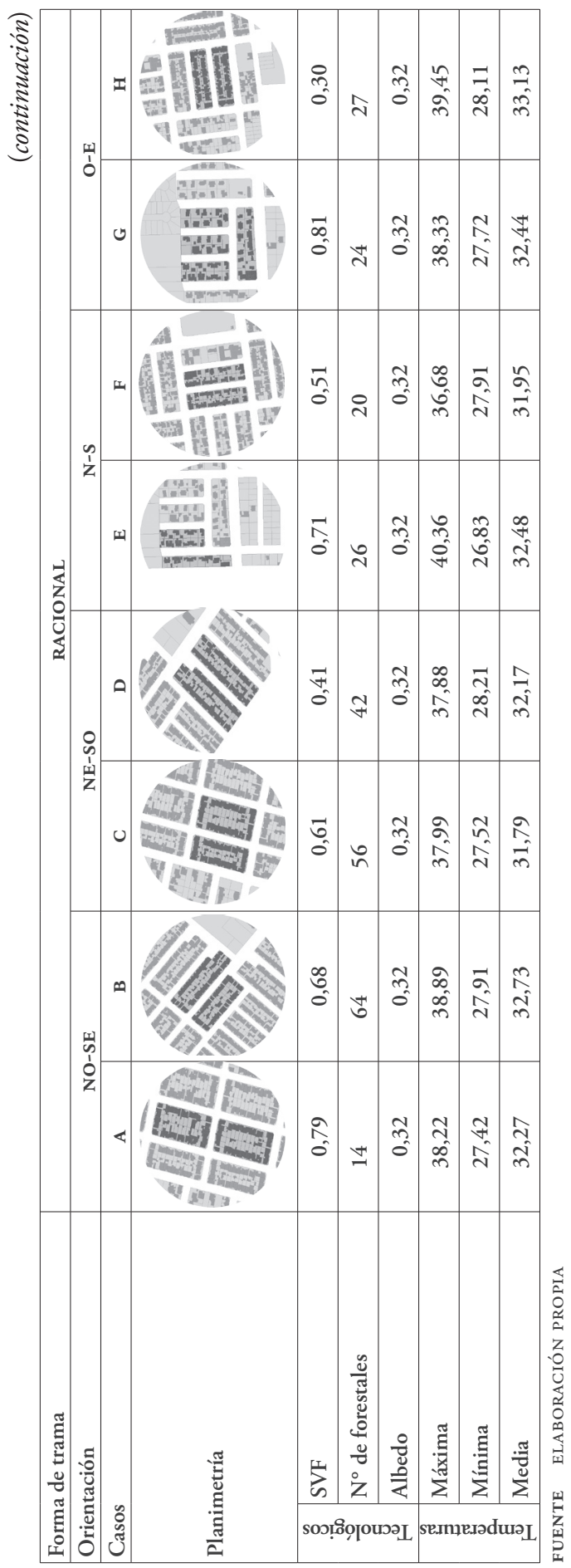




\section{Monitoreo microclimático}

Se caracterizó el comportamiento microclimático de cada uno de los casos evaluados, los cuales fueron monitoreados durante el verano de 2017 (2 al 30 de enero). Los datos se adquirieron mediante campańas de mediciones con sensores fijos, y también se realizaron mediciones itinerantes con estaciones meteorológicas.

En las campañas de mediciones fijas se midió en forma simultánea la temperatura y humedad relativa del aire en cada uno de los casos de estudio, con mediciones cada 15 minutos. Se utilizaron sensores del tipo H08-003-02 ubicados en el centro de cada canal vial, a una altura de 2 metros desde el nivel de la calle (Oke, 2004) dentro de una caja perforada de PVC blanco, con el objeto de evitar la irradiación y asegurar una adecuada circulación de aire.

Las campańas con mediciones móviles tuvieron por objeto monitorear un conjunto más amplio de variables microclimáticas. Para ello se utilizó una estación del tipo HoboWeatherStation ${ }^{\oplus}$, instalada sobre un soporte móvil que permite desplazarla a distintos puntos (se monitorearon cuatro puntos por hora en cada CVU). El periodo de medición con las estaciones móviles comprendió desde las 9:00 hasta las 19:00 horas. En esta campaña se registraron, cada 15 minutos, las variables de temperatura y humedad relativa del aire, temperatura del punto de rocío, velocidad y dirección del viento, presión atmosférica y radiación solar. Simultáneamente se registraron los valores de temperaturas superficiales (vertical-paredes y horizontalcalle y vereda) con un termómetro infrarrojo tipo Fluke 66, y se determinaron los factores de visión de cielo de cada caso mediante el análisis de imágenes hemisféricas digitales. La figura 4 muestra una imagen de los equipamientos utilizados.

\section{FIGURA 4 Equipamientos utilizados para el monitoreo}
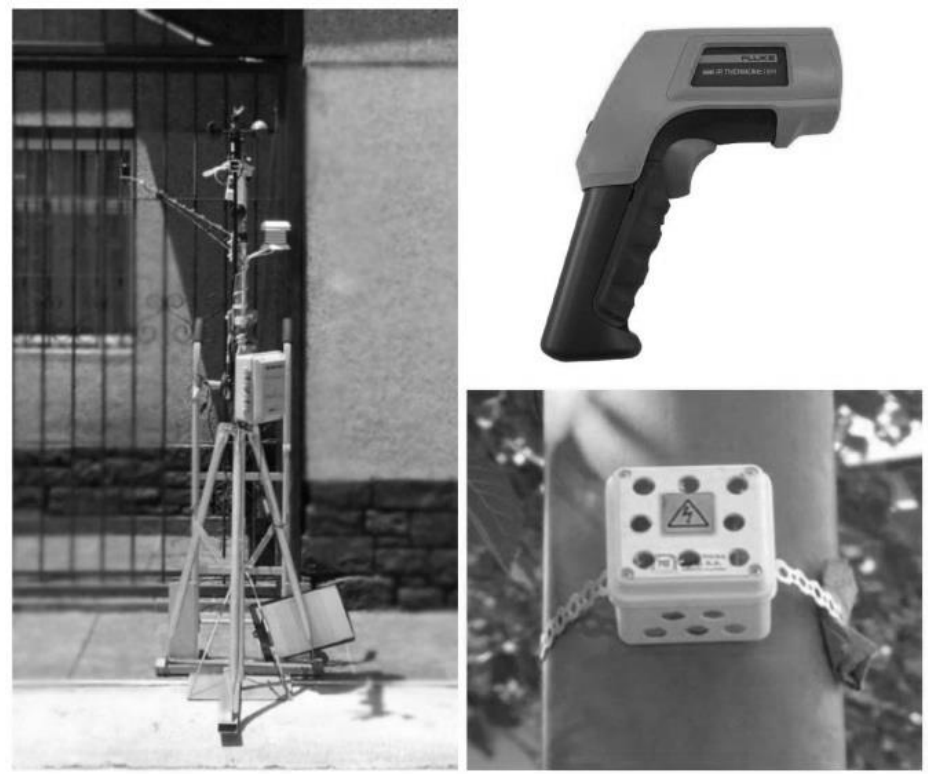

FUENTE FOTOGRAFÍAS DE LAS AUTORAS 


\section{Análisis estadístico}

Para predecir el comportamiento térmico de los diversos casos analizados se utilizaron métodos estadísticos multivariados. Estos métodos son herramientas que estudian el comportamiento de tres o más variables al mismo tiempo y su influencia sobre un determinado fenómeno o variable dependiente. De esta manera buscan detectar las variables que mejor explican el comportamiento de la variable dependiente y descartar las demás. Este procedimiento se efectuó en el trabajo utilizando el software Infostat (Di Rienzo, Casanoves, Balzarini, González, Tablada \& Robledo, 2011).

En primer lugar, se contrastó la normalidad del conjunto de datos mediante el test de Shapiro-Wilks. Este test es considerado uno de los más potentes, sobre todo para muestras pequeñas $(n<30)$. En él se toma como referencia el $\rho$-valor mayor a alfa $(\alpha=0,05)$, para rechazar la hipótesis nula y concluir que los datos siguen una distribución normal. Esto es requisito para aplicar el análisis de datos a través de la matriz de correlaciones y posteriormente desarrollar un análisis de componentes principales (ACP). (Balmaceda, Cantón \& Correa, 2018; Filippín, Ricard \& Larsen, 2013; Ruiz, Sosa, Correa \& Cantón, 2015).

Luego, en una segunda etapa, con el fin de detectar y cuantificar qué variables de la trama urbana muestran relaciones con las temperaturas máxima, mínima y media, se utilizó el coeficiente de correlación de Pearson (r). Los coeficientes de correlación son la expresión numérica que indica el grado de relación lineal existente entre dos variables cuantitativas. El coeficiente de correlación $r$ es un índice cuyos valores absolutos oscilan entre $-1 \mathrm{y}+1$, y cuya magnitud revela el grado de asociación entre las variables. El valor $r=0$ indica que no existe relación entre las variables. Los valores $+/-1$ son indicadores de una correlación perfecta, siendo positiva si al crecer o decrecer $x$, crece o decrece $y$; o negativa si al crecer o decrecer $x$, decrece o crece $y$. En este estudio se considera una relación de magnitud de $+/-0,5$, según corresponda, como significativa. Se utilizó este método para escoger las variables explicativas de mayor importancia, ya que se comparan datos que no son dimensionalmente homogéneos o donde el orden de magnitud de las variables medidas es diferente.

A partir de la selección de variables correlacionadas, se efectuó un análisis de componentes principales (ACP) para temperaturas máxima, mínima y media. El ACP es una técnica multivariante de interdependencia, que permite explicar la mayor parte de la variabilidad total de un conjunto de variables observables, a través de un número menor de componentes o factores comunes no observables. Tiene como objetivo calcular una serie de combinaciones lineales de las variables, de forma que maximicen la varianza de las componentes, con la restricción de que distintas combinaciones lineales sean ortogonales. O, dicho de otro modo, el ACP es una técnica que transforma ciertas variables en otras incorrelacionadas, de media cero, que pueden escribirse como combinaciones lineales de las primeras (llamadas factores o componentes principales), las cuales pueden ordenarse por la magnitud de su varianza. Las primeras $F$ componentes principales bastan para describir, en alto porcentaje, la variabilidad total de las variables originales. Con frecuencia $F$ vale 2 o 3, siendo el primero de ellos el caso más deseable. Aunque todas las variables 
originales están incluidas en la composición de cada componente principal, algunas son más importantes que otras. Las más importantes determinan la naturaleza de cada componente. El ACP es una técnica descriptiva, pero también puede ser utilizado con fines de inferencia.

\section{Resultados}

\section{Comportamiento térmico de las tramas urbanas}

En la figura 5 se presenta el comportamiento de la curva de temperatura del aire para cada uno de los casos monitoreados para un día típico correspondiente al área de estudio. En ella se muestran los comportamientos de los ocho casos monitoreados del día 20 de enero de 2017 . Se seleccionó este día ya que es representativo de las condiciones climática del AMM (día claro, con elevada heliofanía y baja velocidad de vientos y humedad relativa).

El objetivo es identificar qué características presentes en la trama son las que generan menores temperaturas del aire durante el verano, con el fin de diseñar espacios exteriores eficientes, que contribuyan a disminuir los consumos de energía auxiliar para el acondicionamiento térmico de las viviendas, mejorar las condiciones de confort en los espacios públicos de los sectores residenciales y minimizar la isla de calor urbana sobre los sectores de piedemonte de la ciudad, con la consiguiente mitigación de los perjuicios ambientales que ocasiona.

FIgURA 5 | Curva de temperatura del aire para las tramas monitoreadas ubicadas en el piedemonte, día 20 de enero de 2017

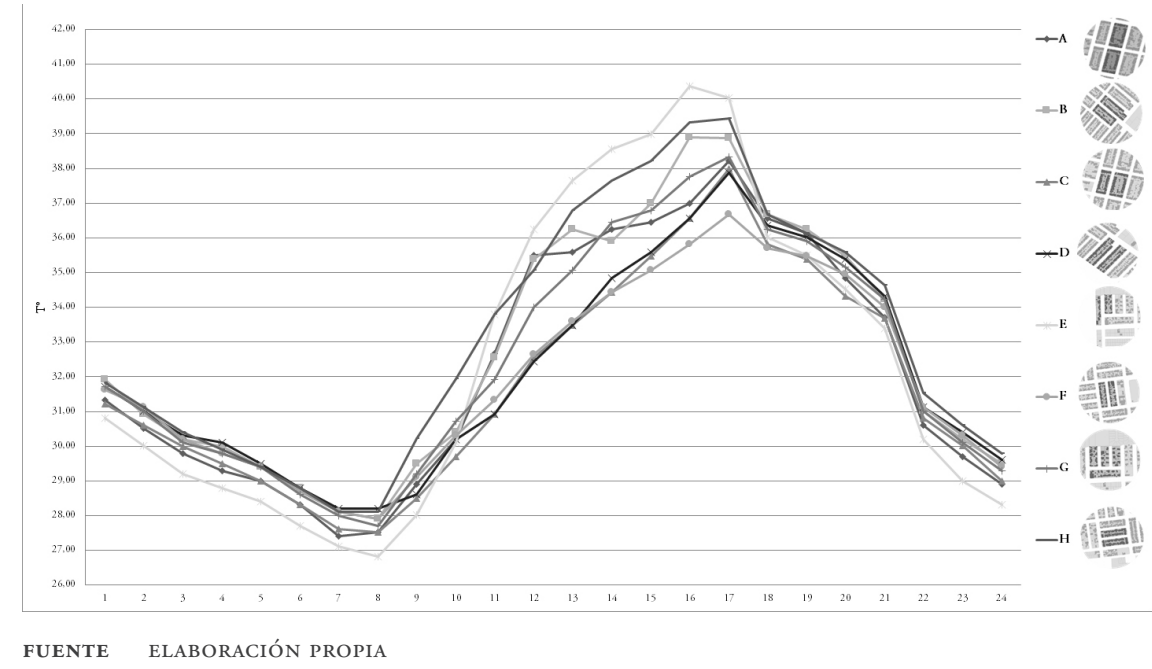

Inicialmente se analizan los casos extremos curvas в y E. Al evaluar y contrastar el comportamiento de ambos, se visualiza que la trama в de orientación $\mathrm{N}$-s, cuya altitud es de 850 m.s.n.m. (curva con seńaladores cuadrados), es la trama, en 
relación al resto, que logra las menores temperaturas durante la noche $\left(26,8{ }^{\circ} \mathrm{C}\right)$ y las mayores temperaturas durante el día $\left(40,3^{\circ} \mathrm{C}\right)$. En cambio, la trama e (curva con marcadores $\mathrm{x}$ ), con la misma orientación pero de altitud de 800 m.s.n.m., posee las menores temperaturas durante el día $\left(36,6{ }^{\circ} \mathrm{C}\right)$; sin embargo, durante la noche se enfría en menor medida, registrando valores de $27,9{ }^{\circ} \mathrm{C}$. La diferencia de las máximas temperaturas es de $3,7^{\circ} \mathrm{C}$ y, en relación con las temperaturas mínimas, es de $1,1^{\circ} \mathrm{C}$.

Si se toma como rango de confort $25^{\circ} \mathrm{C}$ de temperatura durante el día y $22{ }^{\circ} \mathrm{C}$ durante la noche (Givoni, 1991), observamos que durante el día los valores de temperatura del aire para la trama в se alejan $15^{\circ} \mathrm{C}$ de la temperatura de confort; y en la trama E, solo $11^{\circ} \mathrm{C}$. Durante la noche, la trama в se aleja $4{ }^{\circ} \mathrm{C}$ de la temperatura de confort, mientras que la trama $\mathrm{E}$ lo hace en $6^{\circ} \mathrm{C}$. Si tomamos estos valores en relación con el microclima del espacio edilicio conexo, se ve que ambas tramas, con similar orientación morfología y altitud, poseen diferencias muy significativas: la trama в se encuentra $4^{\circ} \mathrm{C}$ más caliente que la $\mathrm{E}$, mientras que durante la noche la E está solo $2{ }^{\circ} \mathrm{C}$ más caliente que la в.

A fin de identificar las variables de diseño responsables de este comportamiento, en la figura 6 se presentan sistematizados los descriptores utilizados para la caracterización de los casos de estudio.

Si comparamos las características de ambas tramas, podemos apreciar cuantitativamente que, aunque ambos casos están orientados de manera similar, la trama в tiene mayor factor de exposición tanto en la mañana como en la noche (porcentaje mayor de factor de apertura de la bóveda). También la relación de conexión entre espacio central de manzana y perimetral es de $3 / 1$ en la trama $\mathrm{B}$, mientras que la $\mathrm{E}$ tiene una relación de 10/1, por lo que hay una gran diferencia en la distribución de áreas abiertas y/o vegetadas dentro de la manzana. Además, se observa que la trama $\mathrm{E}$ posee menor volumen construido (con una diferencia de 5.770,68 $\mathrm{m}^{3}$ ); es decir, hay una diferencia en la inercia térmica y acumulación asociada a la cantidad de masa.

Siguiendo la lógica planteada anteriormente para todos los casos analizados, puede concluirse que, aunque es posible detectar diferencias en el diseño de los casos que posiblemente justifiquen el comportamiento térmico de los mismos de modo cualitativo, es imposible, mediante este método, establecer a priori en qué medida tales diferencias son responsables del comportamiento térmico de la trama. Es por ello que, con el objeto de detectar cuáles de las variables de diseño deben ser controladas para disminuir las temperaturas urbanas de los sectores residenciales localizados en el piedemonte de la ciudad, se recurre al análisis estadístico multivariado. 
FIGURA 6 | Sistematización de descriptores de las tramas в у Е

\begin{tabular}{|c|c|c|c|c|c|}
\hline \multicolumn{3}{|c|}{ CASO B - RACIONAL - ORIENTACIÓN NO-SE } & \multicolumn{3}{|c|}{ CASO E - RACIONAL - ORIENTACIÓN N-S } \\
\hline Posición Geográfica & $\begin{array}{l}32^{\circ} 51^{\prime} 18- \\
68^{\circ} 51^{\prime} 55\end{array}$ & & Posición Geográfica & $\begin{array}{l}32^{\circ} 51^{\prime} 40- \\
68^{\circ} 52^{\prime} 48\end{array}$ & \\
\hline Altitud & 800 & & Altitud & 850 & \\
\hline Azimut & 130 & & Azimut & 181 & \\
\hline Largo & 135 & & Largo & 118 & \\
\hline ANCHO & 16 & & Ancho & 20 & \\
\hline $\mathrm{H} / \mathrm{W}$ & 0,19 & SVF 0,68 & $\mathrm{H} / \mathrm{W}$ & 0,15 & SVF 0,7I \\
\hline Sup.Canal vial urbano & 2160 & & Sup.Canal vial urbano & 2360 & \\
\hline Sup. Total & 8820,16 & & Sup. Total & 7549 & \\
\hline Sup. Total construida & 3123,40 & & Sup. Total construida & 1923,56 & \\
\hline Sup. Total de patios & 3536,76 & & Sup. Total de patios & 3256,56 & \\
\hline Sup. Patios perimetrales & 795,25 & & Sup. Patios perimetrales & 938,38 & \\
\hline Sup. Patios centrales & 2741,51 & & Sup. Patios centrales & 2327,18 & \\
\hline $\begin{array}{l}\text { Rel. Patios centrales y } \\
\text { perimetrales }\end{array}$ & 3,45 & & $\begin{array}{l}\text { Rel. Patios centrales y } \\
\text { perimetrales }\end{array}$ & 2,48 & \\
\hline Compacidad & 1,06 & & Compacidad & 0,76 & \\
\hline Vol. Total construido & 9370,20 & & Vol. Total construido & 5770,68 & \\
\hline $\begin{array}{l}\text { Densidad edilicia } \\
\text { (viv/Ha) }\end{array}$ & 28,34 & & $\begin{array}{l}\text { Densidad edilicia } \\
(\text { viv/Ha) }\end{array}$ & 19,87 & \\
\hline No Viviendas & 25,00 & & No Viviendas & 15 & \\
\hline FOS & 0,45 & & FOS & 0,25 & \\
\hline HORAS SOL & $4: 00$ & & HORAS SOL & $5: 30$ & \\
\hline No de Forestales & 64 & & No de Forestales & 26 & \\
\hline Arbol/m2 & 0,03 & & Arbol $/ \mathrm{m} 2$ & 0,01 & \\
\hline Albedo & 0,32 & & Albedo & 0,32 & \\
\hline Máxima (16:00-17:00) & 38,89 & & Máxima (16:00-17:00) & 40,36 & \\
\hline Mínima (6:00-7:00) & 27,91 & & Mínima (6:00-7:00) & 26,83 & \\
\hline Media & 32,73 & & Media & 32,48 & \\
\hline Amplitud & 10,98 & & Amplitud & 13,53 & \\
\hline
\end{tabular}

FUENTE ELABORACIÓN PROPIA

\section{Análisis estadístico}

Como se explicó en relación con la metodología para detectar, de las variables analizadas, cuáles son las que tienen un comportamiento normal, se realizó en primer lugar el test de Shapiro-Wilks. Los resultados de esa prueba indican que todas las variables son normales, excepto altitud, ancho, relación altura de viviendas con ancho de canal y compacidad, por lo que fueron descartadas del análisis.

Se propone realizar un ACP con el objetivo observar el comportamiento de la variable que se quieres analizar (en este caso, temperaturas máxima, mínima y media) en relación con el comportamiento de distintas variables que representan los componentes del espacio urbano que se busca vincular (componente morfológico, componente geomorfológico y componente tecnológico). Dado que el método de componentes principales es considerado un método de reducción de datos, se 
utiliza para reducir la dimensión del número de variables que inicialmente se han considerado y determinar un nuevo conjunto de variables, las componentes principales, que gozan de la ventaja de estar incorrelacionadas entre sí y que, además, pueden ordenarse de acuerdo con la información que llevan incorporada.

Para ello, inicialmente se utilizaron todas las variables analizadas que muestran comportamiento normal. Los resultados del ACP, para el caso de las temperaturas máximas, se muestran en la tabla 1 . Se observa la matriz de correlaciones, donde se resaltan en negrita las variables que tienen alta correlación con significancia estadística; es decir, $p<0,05$. A partir del análisis de la matriz de correlaciones (tabla 1), se aprecia que la variable con mayor correlación a la temperatura máxima es el fos (factor de ocupación de suelo - indicador morfológico).

Existen varios contrastes que pueden realizarse para evaluar si el modelo factorial (o la extracción de los factores) en su conjunto es significativo. El test KMO (Kaiser, Meyer y Olkin) relaciona los coeficientes de correlación, rjh, observados entre las variables $X j$ y $X h$, y ajh, con los coeficientes de correlación parcial entre las variables $X j$ y $X h$. Cuanto más cerca de 1 tenga el valor obtenido del test кмо, la relación entre las variables es más alta. Se recomienda no aplicar ACP para valores de KMO < 0,5 . Por otra parte, la prueba de esfericidad de Bartlett evalúa la aplicabilidad del análisis factorial de las variables estudiadas. El modelo es significativo (aceptamos la hipótesis nula, $H O$ ) cuando se puede aplicar el análisis factorial (De la Fuente Fernández, 2011).

Sin embargo, de los resultados de la tabla 1, vemos que la prueba de esfericidad de Barlett no se puede aplicar debido a la existencia de colinealidad entre las variables consideradas; y, por otra parte, el valor de KMO es levemente inferior a 0,5 , restándole significancia estadística -es decir, valor- a los resultados de nuestro análisis. 


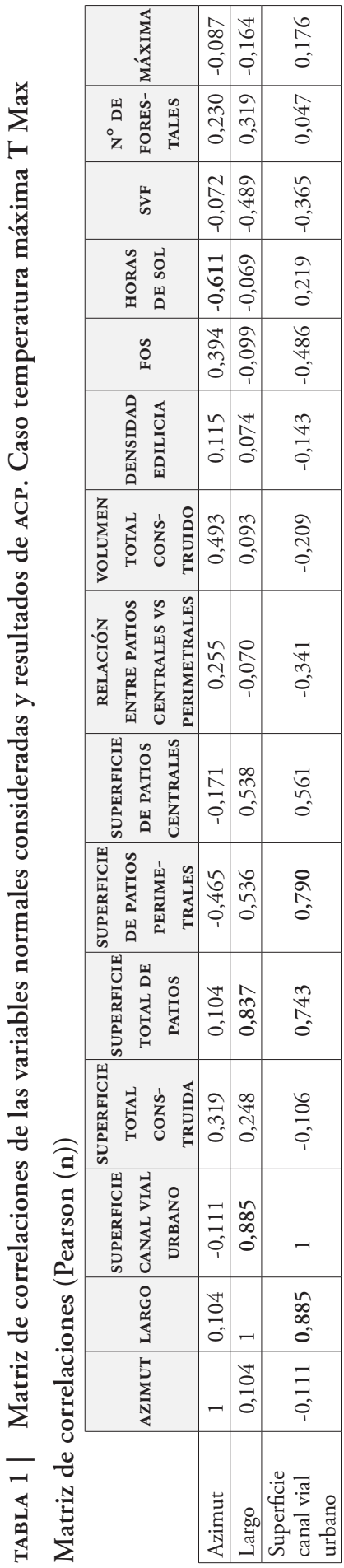

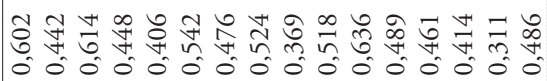

齿

离

:

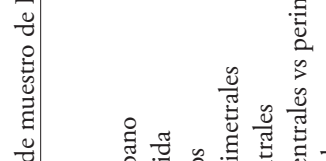

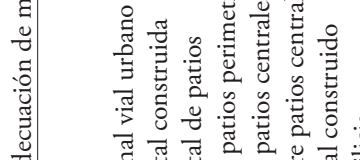

产

ष

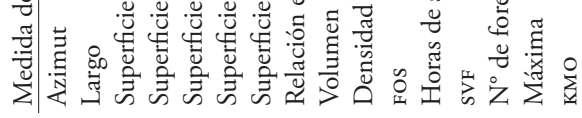

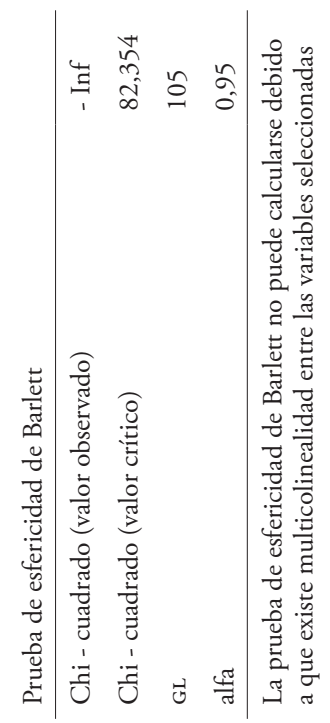




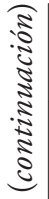

\begin{tabular}{|c|c|c|c|c|c|c|c|c|c|c|c|c|}
\hline$\sum_{\substack{x \\
\vdots}}^{x}$ & 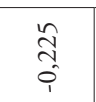 & $\begin{array}{l}\text { ָे } \\
\text { î }\end{array}$ & $\begin{array}{l}\text { సे } \\
\text { ô }\end{array}$ & 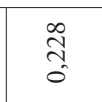 & 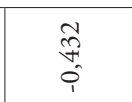 & $\begin{array}{l}\infty \\
\stackrel{0}{0} \\
0 \\
0 \\
1\end{array}$ & $\begin{array}{l}\hat{n} \\
\hat{i}\end{array}$ & $\mid \begin{array}{c}0 \\
\infty \\
1 \\
0 \\
1 \\
1\end{array}$ & \begin{tabular}{l|l}
$\infty$ \\
0 \\
0 \\
0 \\
1
\end{tabular} & $\begin{array}{l}\hat{\alpha} \\
\hat{0} \\
\hat{0}\end{array}$ & $\begin{array}{l}0 \\
0 \\
0 \\
0\end{array}$ & - \\
\hline 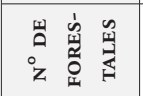 & $\begin{array}{l}\stackrel{?}{2} \\
\hat{0}\end{array}$ & \begin{tabular}{l}
0 \\
$\infty$ \\
\multirow{1}{0}{} \\
0
\end{tabular} & $\begin{array}{l}\text { ते } \\
\text { î }\end{array}$ & $\begin{array}{l}\vec{F} \\
\overrightarrow{0}\end{array}$ & $\begin{array}{l}\tilde{n} \\
0 \\
0 \\
i\end{array}$ & $\begin{array}{l}\mathbb{J} \\
\text { ర } \\
0\end{array}$ & 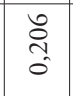 & \begin{tabular}{|l|}
$\infty$ \\
$\stackrel{1}{a}$ \\
0 \\
0
\end{tabular} & $\begin{array}{l}n \\
0 \\
0 \\
1\end{array}$ & $\begin{array}{c}-1 \\
0 \\
0 \\
1\end{array}$ & - & $\begin{array}{l}0 \\
0 \\
0 \\
0\end{array}$ \\
\hline$\sum_{n}^{10}$ & 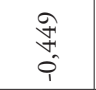 & $\begin{array}{l}n \\
0 \\
0 \\
1\end{array}$ & $\begin{array}{l}\infty \\
\stackrel{2}{0} \\
0\end{array}$ & $\begin{array}{c}\infty \\
\infty \\
0 \\
i\end{array}$ & $\begin{array}{l}\hat{0} \\
\text { S. } \\
0 \\
0\end{array}$ & $\begin{array}{l}n \\
\hat{n} \\
0\end{array}$ & $\begin{array}{l}n \\
\infty \\
\infty \\
0\end{array}$ & $\mid$\begin{tabular}{c}
0 \\
\multirow{+}{+}{} \\
0 \\
1
\end{tabular} & $\begin{array}{l}n \\
\infty \\
2 \\
0\end{array}$ & - & $\begin{array}{l}\overrightarrow{0} \\
\stackrel{0}{0} \\
i\end{array}$ & $\hat{\sigma}$ \\
\hline 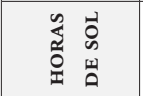 & $\begin{array}{l}\text { बे } \\
\text { i }\end{array}$ & $\begin{array}{l}0 \\
0 \\
0 \\
0\end{array}$ & $\begin{array}{l}8 \\
0 \\
0 \\
1\end{array}$ & 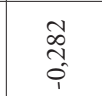 & $\begin{array}{l}\stackrel{2}{ } \\
\hat{i} \\
\hat{i}\end{array}$ & $\begin{array}{l}\text { oे } \\
\text { +े } \\
\text { co }\end{array}$ & $\begin{array}{l}R \\
\hat{G} \\
0 \\
i\end{array}$ & $\begin{array}{l}-\vec{T} \\
0 \\
0 \\
i\end{array}$ & - & $\begin{array}{l}n \\
\infty \\
0 \\
0 \\
0\end{array}$ & 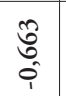 & $\begin{array}{l}\infty \\
0 \\
0 \\
0 \\
1\end{array}$ \\
\hline : & $\begin{array}{l}\text { R̂. } \\
\text { O } \\
0\end{array}$ & 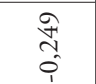 & $\begin{array}{l}\stackrel{8}{\infty} \\
\hat{0} \\
i\end{array}$ & $\begin{array}{l}\vec{m} \\
\overrightarrow{0}\end{array}$ & $\begin{array}{l}\infty \\
\infty \\
\infty \\
0\end{array}$ & 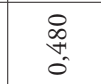 & $\begin{array}{l}\mathbb{7} \\
\hat{0}\end{array}$ & - & $\begin{array}{l}\overrightarrow{0} \\
0 \\
i\end{array}$ & $\begin{array}{l}2 \\
\vdots \\
+ \\
1\end{array}$ & \begin{tabular}{l}
$\infty$ \\
\multirow{1}{*}{} \\
0
\end{tabular} & $\begin{array}{l}0 \\
2 \\
n \\
0 \\
1\end{array}$ \\
\hline 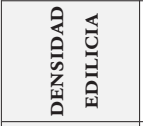 & $\begin{array}{l}\stackrel{+}{+} \\
\stackrel{+}{*} \\
0\end{array}$ & $\begin{array}{l}\hat{\varkappa} \\
\hat{0} \\
i\end{array}$ & 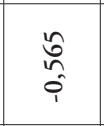 & $\begin{array}{l}\text { ठㅇ } \\
0\end{array}$ & 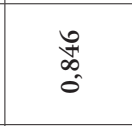 & 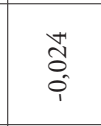 & - & $\begin{array}{c}7 \\
7 \\
0 \\
0\end{array}$ & $\begin{array}{l}0 \\
\mathbb{6} \\
0 \\
i\end{array}$ & $\begin{array}{l}n \\
0 \\
\infty \\
0 \\
1\end{array}$ & 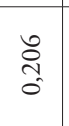 & $\begin{array}{l}2 \\
0 \\
0 \\
1\end{array}$ \\
\hline 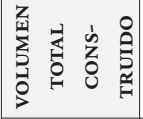 & $\begin{array}{l}\hat{0} \\
\cong \\
0\end{array}$ & $\begin{array}{l}\tilde{3} \\
\text { I. } \\
0\end{array}$ & $\begin{array}{l}\hat{0} \\
\hat{0}\end{array}$ & $\begin{array}{l}\stackrel{N}{\sim} \\
\text { î } \\
\stackrel{i}{1}\end{array}$ & \begin{tabular}{l}
\multirow{2}{0}{} \\
î \\
$i$
\end{tabular} & - & 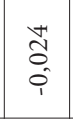 & $\left|\begin{array}{c}0 \\
\infty \\
+ \\
0 \\
0\end{array}\right|$ & $\begin{array}{l}2 \\
\text { के } \\
+ \\
0 \\
1\end{array}$ & $\begin{array}{l}\hat{n} \\
\hat{0} \\
0\end{array}$ & 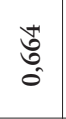 & $\begin{array}{l}\infty \\
0 \\
0 \\
0 \\
1 \\
1\end{array}$ \\
\hline 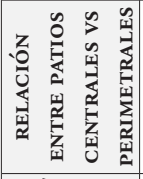 & $\begin{array}{l}\hat{\sigma} \\
\hat{0}\end{array}$ & $\begin{array}{l}\overrightarrow{\widetilde{N}} \\
\hat{\widetilde{2}} \\
\hat{i}_{1}\end{array}$ & $\begin{array}{l}\sqrt{\infty} \\
\stackrel{1}{0} \\
\hat{i}\end{array}$ & $\frac{m}{n}$ & - & 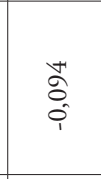 & $\begin{array}{l}0 \\
+ \\
\infty \\
0 \\
0\end{array}$ & $\mid \begin{array}{c}\infty \\
0 \\
\infty \\
0 \\
0\end{array}$ & 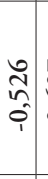 & $\begin{array}{l}\hat{0} \\
0 \\
\hat{i}\end{array}$ & $\begin{array}{l}\tilde{n} \\
\text { Ð } \\
0 \\
i\end{array}$ & 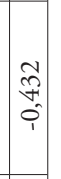 \\
\hline 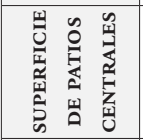 & $\begin{array}{l}\text { Iี } \\
\text { ô }\end{array}$ & $\begin{array}{l}\hat{\sim} \\
\hat{0}\end{array}$ & $\begin{array}{l}\stackrel{\infty}{2} \\
0\end{array}$ & - & $\frac{m}{2}$ & $\begin{array}{l}\text { f } \\
\text { î } \\
i\end{array}$ & $\begin{array}{l}\text { హ్ర } \\
0\end{array}$ & $\begin{array}{l}\vec{n} \\
0\end{array}$ & 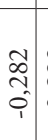 & $\begin{array}{l}\infty \\
\infty \\
\hat{\rho} \\
1\end{array}$ & $\begin{array}{l}\vec{\nabla} \\
\overrightarrow{0}\end{array}$ & 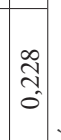 \\
\hline 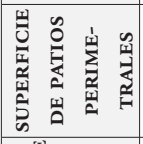 & $\begin{array}{l}0 \\
\text { ñ } \\
\text { ? }\end{array}$ & $\begin{array}{l}\hat{w} \\
\hat{n} \\
\hat{0}\end{array}$ & - & $\begin{array}{l}\stackrel{\infty}{=} \\
0\end{array}$ & $\begin{array}{l}\stackrel{+}{\infty} \\
\stackrel{0}{0} \\
i\end{array}$ & $\begin{array}{l}\hat{0} \\
\hat{0}\end{array}$ & 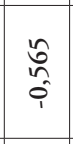 & $\mid \begin{array}{c}0 \\
\infty \\
0 \\
0 \\
1\end{array}$ & $\begin{array}{l}8 \\
0 \\
0 \\
1\end{array}$ & $\left|\begin{array}{l}\infty \\
0 \\
0 \\
0\end{array}\right|$ & $\begin{array}{l}\text { ते } \\
\text { iे } \\
\text { i }\end{array}$ & 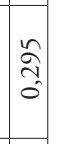 \\
\hline 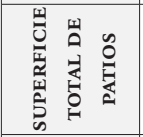 & $\begin{array}{l}\text { ڤे } \\
\text { ڤे } \\
0\end{array}$ & - & $\begin{array}{l}n \\
\infty \\
0 \\
0\end{array}$ & $\begin{array}{l}\hat{\imath} \\
\hat{0}\end{array}$ & $\begin{array}{l}\overrightarrow{\widetilde{N}} \\
\tilde{0} \\
\hat{i}\end{array}$ & $\begin{array}{l}\text { ్ֶర } \\
\text { ñ. } \\
0\end{array}$ & $\begin{array}{l}\text { ڤิ } \\
\hat{0} \\
i\end{array}$ & 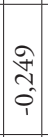 & $\begin{array}{l}0 \\
0 \\
0 \\
0\end{array}$ & 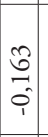 & $\begin{array}{l}0 \\
\infty \\
+ \\
0 \\
0\end{array}$ & $\mid \begin{array}{l}0 \\
0 \\
0 \\
0 \\
1\end{array}$ \\
\hline 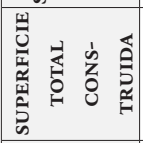 & - & $\begin{array}{l}\text { ¿ } \\
\text { ले }\end{array}$ & $\begin{array}{l}0 \\
\text { ñ } \\
\text { ?े }\end{array}$ & $\begin{array}{l}\underset{7}{4} \\
\text { Ȯ }\end{array}$ & $\begin{array}{c}\hat{\sigma} \\
\hat{\sigma}\end{array}$ & $\begin{array}{l}\hat{0} \\
\stackrel{2}{0} \\
0\end{array}$ & 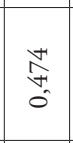 & $\begin{array}{l}R \\
6 \\
0 \\
0\end{array}$ & 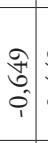 & $\left|\begin{array}{l}\mathfrak{f} \\
\mathbb{f} \\
0 \\
1\end{array}\right|$ & 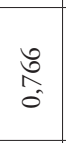 & 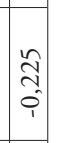 \\
\hline 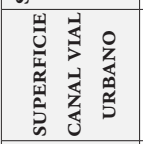 & $\begin{array}{l}0 \\
\stackrel{0}{0} \\
i\end{array}$ & $\frac{\stackrel{2}{+}}{\hat{0}}$ & $\begin{array}{l}\stackrel{2}{\hat{\imath}} \\
0\end{array}$ & $\begin{array}{l}\bar{J} \\
\cong \\
0\end{array}$ & $\begin{array}{l}\vec{F} \\
\text { के } \\
\hat{i}\end{array}$ & 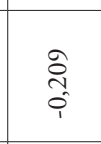 & $\frac{\stackrel{P}{+}}{\stackrel{p}{i}}$ & $\begin{array}{l}0 \\
\infty \\
0 \\
0 \\
0 \\
1\end{array}$ & 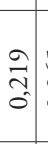 & $\mid \begin{array}{l}n \\
0 \\
0 \\
0 \\
1\end{array}$ & $\begin{array}{l}\hat{y} \\
\text { - } \\
0\end{array}$ & $\begin{array}{c}0 \\
\\
0 \\
0\end{array}$ \\
\hline 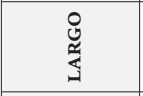 & $\begin{array}{l}\infty \\
\stackrel{\infty}{1} \\
0\end{array}$ & $\begin{array}{l}\hat{⿵} \\
\infty \\
0 \\
0\end{array}$ & $\begin{array}{l}\text { D. } \\
\approx \\
\hat{0}\end{array}$ & $\begin{array}{l}\infty \\
\approx \\
\hat{0}\end{array}$ & $\begin{array}{l}\text { R } \\
0 \\
0 \\
1\end{array}$ & $\begin{array}{l}m \\
\tilde{\delta} \\
0\end{array}$ & \begin{tabular}{l}
+ \\
\multirow{2}{0}{} \\
0
\end{tabular} & $\begin{array}{l}\hat{\alpha} \\
\hat{\sigma} \\
\hat{0} \\
i\end{array}$ & $\begin{array}{l}a \\
\dot{0} \\
0 \\
1\end{array}$ & \begin{tabular}{|c|} 
\\
0 \\
0 \\
0 \\
1 \\
1 \\
\end{tabular} & $\begin{array}{c}\stackrel{\vec{े}}{2} \\
0\end{array}$ & 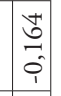 \\
\hline $\begin{array}{l}5 \\
5 \\
\\
\end{array}$ & $\begin{array}{l}2 \\
\hat{0}\end{array}$ & $\begin{array}{l}\text { tr } \\
\stackrel{0}{0}\end{array}$ & 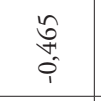 & $\underset{i}{\vec{i}}$ & $\begin{array}{l}\tilde{n} \\
\text { ֶิ } \\
0\end{array}$ & $\underset{0}{\stackrel{m}{q}}$ & $\stackrel{n}{\Rightarrow}$ & $\begin{array}{c}+1 \\
\tilde{2} \\
0\end{array}$ & $\begin{array}{l}7 \\
\overrightarrow{0} \\
0 \\
1\end{array}$ & $\begin{array}{l}\tilde{N} \\
\hat{0} \\
0 \\
1 \\
\end{array}$ & 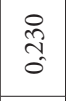 & $\begin{array}{l}\hat{0} \\
0 \\
0 \\
1 \\
\end{array}$ \\
\hline & 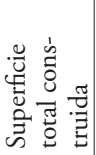 & 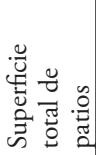 & 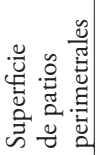 & 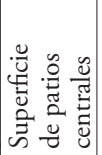 & 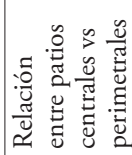 & 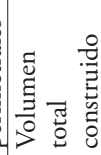 & 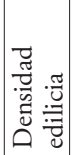 & $\mid \begin{array}{l}0 \\
0 \\
1\end{array}$ & 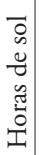 & 岕 & 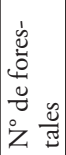 & : \\
\hline
\end{tabular}


Cuando se repite el proceso anterior, se observa un comportamiento semejante en cuanto a las temperaturas mínima y media. En ninguno de estos casos puede realizarse la prueba de esfericidad de Barlett, debido a la existencia de colinealidad entre las variables. Los valores de кMO son 0,512 para el caso de la matriz que contiene la temperatura mínima y 0,475 para el análisis que contiene la temperatura media. El análisis de la matriz de correlaciones muestra que: la temperatura mínima correlaciona significativamente con el largo del canal vial $(0,515)$, la superficie de patios centrales $(0,582)$, la densidad edilicia $(0,562)$ y el SvF $(-0,650)$; es decir, presenta máxima correlación con un indicador morfotecnológico, dos indicadores morfológicos que refieren a la configuración de la manzana y un indicador morfológico que refiere a la trama. Por su parte, la temperatura media correlaciona con el azimut $(-0,565)$, la superficie de patios centrales $(0,590)$ y el volumen total construido $(-0,702)$; es decir, con un indicador geomorfológico y dos indicadores morfológicos que refieren a la estructura de la manzana.

Posteriormente, se decide explorar para cada una de las situaciones analizadas (temperaturas máxima, mínima y media) las combinaciones de variables que le den solidez estadística a este análisis. Las tablas 2, 3 y 4 muestran el conjunto de variables para cada una de las situaciones examinadas, lo que permite realizar un $\mathrm{ACP}$, cumpliendo con la condición de que el análisis factorial sea significativo en su conjunto.

Las combinaciones de variables que representan esta condición para el análisis del comportamiento de la temperatura máxima son: azimut, svF, relación superficie de patios centrales y perimetrales, fos y horas de sol (tabla 2).

El análisis cumple con la prueba de esfericidad de Barlett, y el KMO es 0,612. Los resultados indican que los dos primeros factores cuyo autovalor es mayor que uno véase gráfico de segmentación y valores propios en la tabla 2- podrían explicar hasta el $73 \%$ del comportamiento del conjunto analizado. Si observamos los vectores propios y el comportamiento de los cosenos cuadrados de las variables, vemos que el primer factor está formado por la relación entre dos indicadores morfológicos de la manzana -Fos (factor de ocupación) y la relación de patios centrales y perimetrales- en sentido inverso al comportamiento de la temperatura máxima; es decir, que si sube la ocupación de suelo y la apertura del centro de la manzana, la temperatura máxima del canal vial disminuye, mientras que, también, depende en sentido directo de las horas de exposición solar de la trama. En su conjunto, este factor hace referencia a los patrones de ocupación de la manzana, indicando que una mayor ocupación y concentración del espacio abierto en el centro de la misma incrementan el efecto de la masa térmica del espacio construido, disminuyendo la magnitud de la temperatura máxima, del mismo modo que sucede con el control de la exposición solar de la trama.

El segundo factor explicativo de la temperatura máxima es el azimut, es decir, la relación entre la orientación de la trama y el recorrido solar, el cual indica que a medida que la orientación se encuentra más cercana al oeste, mayor es la temperatura máxima, puesto que el valor de azimut se considera igual a 0 en el sur.

El objetivo último de este trabajo es explicar el comportamiento térmico de los casos analizados, para proponer adecuados criterios de diseńo. El análisis pone 
de manifiesto que un diseño bioclimático de áreas de piedemonte que pretenda controlar las temperaturas máximas, debe propender a determinar condiciones óptimas de sellamiento de suelo en la manzana. Para ello debe conciliar el control de riesgo aluvional y la fragilidad ambiental de estas áreas, lo que demanda minimizar el sellamiento de suelos, con el efecto de la inercia térmica del espacio construido, que es benéfica para la disminución de las temperaturas máximas. Por otra parte, es necesario controlar la exposición solar, pero además distribuir adecuadamente los espacios libres interiores.

Lo anterior también se ve reflejado por el comportamiento de la densidad edilicia, debido a que otra combinación posible de variables, que permite un ACP con significancia estadística del comportamiento de las temperaturas máximas, es el conjunto compuesto por superficie de canal vial, densidad edilicia, relación entre superficie de patios centrales y perimetrales y svf. El conjunto cumple con la prueba de esfericidad de Barlett y tiene un кмо de 0,51. Indica que mediante dos factores cuyo autovalor es superior a uno, se podría explicar el 80\% del comportamiento del conjunto. El primer factor agrupa, con un comportamiento inverso, la densidad edilicia y la relación de patios centrales y perimetrales, indicando que la temperatura máxima disminuye cuando ambas variables se incrementan; y en sentido directo al SVF, indicando que a mayor factor de visión de cielo, mayor es la temperatura máxima. El segundo factor está explicado enteramente y en sentido directo por la superficie de canal vial, y expresa que cuando esta aumenta, la temperatura máxima también lo hace. Al igual que en el análisis anterior, el comportamiento del conjunto se explica mediante el efecto de dos indicadores morfológicos vinculados a la distribución de la edificación en la manzana, y el efecto de un indicador morfotecnológico como el svf, que muestra el efecto de la exposición solar. Por su parte, el segundo factor hace alusión a la configuración de la trama.

Se observa que si la densidad edilicia aumenta la temperatura máxima disminuye, lo que posibilitaría incrementar la superficie construida sin necesidad de aumentar el fos, ya que aumentar el sellamiento de suelos en áreas de piedemonte está contraindicado, pues perjudica el porcentaje de infiltración, generando el riesgo aluvional. Finalmente, se observa que la variable svf, que combina el efecto del perfil urbano y la forma de la vegetación sobre las posibilidades de visión de bóveda celeste, resulta ser una variable más explicativa del comportamiento de la temperatura que el número de árboles. Esto pone en manifiesto la importancia del adecuado diseño y selección de la forestación en relación con la geometría del canal vial.

En el caso de las temperaturas mínimas, las combinaciones de variables que permiten un adecuado ACP son: superficie del canal vial, relación superficie de patios centrales y perimetrales, Fos, densidad edilicia y svf (tabla 3). 
TABLa 2 Análisis de componentes principales. Caso de las temperaturas máximas

Prueba de esfericidad de Bartlett

\begin{tabular}{|l|l|}
\hline Chi-cuadrado (valor observado) & 15,295 \\
\hline Chi-cuadrado (valor crítico) & 7,261 \\
\hline GL & 15 \\
\hline valor-p (bilateral) & 0 \\
\hline alfa & 0,95 \\
\hline
\end{tabular}

Medida de adecuación de muestreo de Kaiser-Meyer-Olkin

\begin{tabular}{|l|c|}
\hline Azimut & 0,638 \\
\hline SVF & 0,764 \\
\hline Relación entre patios centrales y perimetrales & 0,809 \\
\hline FOS & 0,628 \\
\hline Horas de sol & 0,525 \\
\hline Máxima & 0,352 \\
\hline KMO & 0,612 \\
\hline
\end{tabular}

Análisis de Componentes Principales

Valores propios:

\begin{tabular}{l|c|c|c|c|c|c}
\cline { 2 - 6 } & FI & F2 & F3 & F4 & F5 & F6 \\
\hline Valor propio & 3,120 & 1,266 & 0,957 & 0,361 & 0,207 & 0,090 \\
\hline Variabilidad (\%) & 51,995 & 21,099 & 15,944 & 6,011 & 3,444 & 1,507 \\
\hline$\%$ acumulado & 51,995 & 73,094 & 89,039 & 95,049 & 98,493 & 100,000
\end{tabular}

FUENTE ELABORACIÓN PROPIA

El análisis cumple con la prueba de esfericidad de Barlett, y el KMO es 0,671. Los resultados indican que los dos primeros factores cuyo autovalor es mayor que uno -véase gráfico de segmentación y valores propios en la tabla 3- podrían explicar hasta el $87 \%$ del comportamiento del conjunto. Si observamos los vectores propios y el comportamiento de los cosenos cuadrados de las variables, vemos que el primer factor está formado por la relación entre tres indicadores morfológicos de la manzana-FOs, densidad edilicia y relación de patios centrales y perimetrales- en sentido directo a la temperatura mínima; es decir, que si suben la ocupación de suelo, la densidad edilicia y la apertura del centro de la manzana, la temperatura mínima del conjunto aumenta, mientras que también depende inversamente del svf, indicando que si se incrementan las posibilidades de visión de cielo de la trama, la temperatura mínima disminuye. En su conjunto, este factor hace referencia a los patrones de ocupación de la manzana, indicando que una mayor ocupación y concentración del espacio abierto en el centro de la misma incrementan el efecto de la acumulación de calor, perjudicando las posibilidades de enfriamiento del espacio. Del mismo modo, una mayor visión de cielo en la disposición y distribución de las superficies edificadas permitiría conseguir mejores condiciones de enfriamiento nocturno.

El segundo factor explicativo de la temperatura mínima es la superficie de canal vial, del mismo modo que sucedía para el caso de la máxima, en que mayor superficie de canal vial significa una trama urbana más abierta, con mayor condición 
de exposición solar y acumulación de calor, lo que perjudica las posibilidades de enfriamiento nocturno de la trama.

El análisis de la respuesta estadística indica que, respecto a las posibilidades de enfriamiento de los sectores residenciales localizados en el piedemonte, las mismas mejoran cuando se incrementan las posibilidades de visión de bóveda celeste. Además, mejoran con la disminución de la superficie construida y la densidad edilicia, y evitando la concentración de las áreas libres en el centro de la manzana; es decir, distribuyendo la superficie libre más hacia la periferia de la manzana que hacia el centro de la misma.

Se observa que los parámetros morfológicos relacionados a la intensidad de ocupación de suelo en la manzana y la distribución del espacio abierto en el interior de la misma influyen de forma opuesta en la afectación de las temperaturas máximas y mínimas. Esto demanda la exploración de soluciones de compromiso y la determinación de rangos de ocupación y distribución del espacio libre que permitan controlar la magnitud de las temperaturas máximas durante el día, sin afectar las posibilidades de enfriamiento nocturno de las urbanizaciones, incrementando el efecto de isla de calor urbana. Sin embargo, es claro que tanto para disminuir las temperaturas máximas durante el día como las mínimas durante la noche, es preciso controlar la superficie de canal vial, propiciando un diseño que posibilite un correcto desempeño funcional, evitando para ello las tramas urbanas excesivamente abiertas.

TABLA 3 Análisis de componentes principales. Caso de las temperaturas mínimas

Prueba de esfericidad de Bartlett

\begin{tabular}{|l|l|}
\hline Chi-cuadrado (valor observado) & 24,461 \\
\hline Chi-cuadrado (valor crítico) & 7,261 \\
\hline GL & 15 \\
\hline valor-p (bilateral) & 0,058 \\
\hline alfa & 0,95 \\
\hline
\end{tabular}

Medida de adecuación de muestreo de Kaiser-Meyer-Olkin

\begin{tabular}{|l|l|}
\hline Superficie de canal vial urbano & 0,296 \\
\hline Relación entre patios centrales y perimetrales & 0,885 \\
\hline Densidad edilicia & 0,718 \\
\hline FOS & 0,745 \\
\hline SVF & 0,578 \\
\hline Mínimo & 0,811 \\
\hline KMO & 0,671 \\
\hline
\end{tabular}

Análisis de Componentes Principales

Valores propios:

\begin{tabular}{|l|c|c|c|c|c|c}
\cline { 2 - 6 } & FI & F2 & F3 & F4 & F5 & F6 \\
\hline Valor propio & 3,593 & 1,626 & 0,415 & 0,187 & 0,132 & 0,047 \\
\hline Variabilidad (\%) & 59,890 & 27,095 & 6,919 & 3,114 & 2,195 & 0,788 \\
\hline$\%$ acumulado & 59,890 & 86,985 & 93,903 & 97,017 & 99,212 & 100,000
\end{tabular}

FUENTE ELABORACIÓN PROPIA 
Por último, en la tabla 4 se observa el conjunto de variables consideradas para el análisis del comportamiento de la temperatura media: superficie del canal vial, svf, relación superficie de patios centrales y perimetrales, Fos, densidad edilicia y horas de sol.

El análisis cumple con la prueba de esfericidad de Barlett, y el кмо es 0,542. Los resultados indican que los dos primeros factores cuyo autovalor es mayor que uno véase gráfico de segmentación y valores propios en la tabla 2- podrían explicar hasta el $79 \%$ del comportamiento del conjunto analizado. Si observamos los vectores propios y el comportamiento de los cosenos cuadrados de las variables, vemos que el primer factor está formado por la relación entre tres indicadores morfológicos de la manzana -factor de ocupación, densidad edilicia y relación de patios centrales y perimetrales-, en sentido inverso al comportamiento de la temperatura máxima; es decir, que si sube la ocupación de suelo, la densidad edilicia y la apertura del centro de la manzana, la temperatura media del canal vial disminuye. Por otra parte, la temperatura media también depende en sentido directo de las horas de exposición solar y el factor de visión de cielo; esto es, si ambos indicadores se incrementan, la temperatura media también lo hace, en referencia a la condición de exposición solar del conjunto. Al igual que para el caso de la temperatura máxima, este factor hace referencia a los patrones de ocupación de la manzana, indicando que una mayor ocupación, densidad edilicia y concentración del espacio abierto en el centro de la misma, incrementan el efecto de la masa térmica del espacio construido, disminuyendo la magnitud de la temperatura media, del mismo modo que sucede con el control de la exposición solar: a mayor control -menor svf y horas de sol-, menor temperatura media.

El segundo factor explicativo de la temperatura media es la superficie de canal vial: a medida que esta aumenta también lo hace la temperatura media, reforzando lo dicho para los casos de control de temperatura máxima y mínima; esto es, que para disminuir la temperatura media de las urbanizaciones en el piedemonte, es necesario evitar tramas urbanas excesivamente abiertas.

TABLA 4 Análisis de componentes principales. Caso de las temperaturas medias Prueba de esfericidad de Bartlett

\begin{tabular}{|l|l|}
\hline Chi-cuadrado (valor observado) & 35,416 \\
\hline Chi-cuadrado (valor crítico) & 11,591 \\
\hline GL & 21 \\
\hline valor-p (bilateral) & 0,025 \\
\hline
\end{tabular}

Medida de adecuación de muestreo de Kaiser-Meyer-Olkin

\begin{tabular}{|l|c|}
\hline Superficie de canal vial urbano & 0,343 \\
\hline Relación entre patios centrales y perimetrales & 0,806 \\
\hline Densidad edilicia & 0,564 \\
\hline Fos & 0,546 \\
\hline Horas de sol & 0,685 \\
\hline SVF & 0,596 \\
\hline Media & 0,199 \\
\hline Kмо & 0,542 \\
\hline
\end{tabular}


Análisis de Componentes Principales

Valores propios:

\begin{tabular}{l|c|c|c|c|c|c|c}
\cline { 2 - 8 } & FI & F2 & F3 & F4 & F5 & F6 & F7 \\
\hline Valor propio & 3,703 & 1,829 & 0,752 & 0,536 & 0,139 & 0,031 & 0,008 \\
\hline Variabilidad (\%) & 52,904 & 26,133 & 10,749 & 7,658 & 1,991 & 0,449 & 0,116 \\
\hline \% acumulado & 52,904 & 79,037 & 89,786 & 97,444 & 99,435 & 99,884 & 100,00
\end{tabular}

FUENTE ELABORACIÓN PROPIA

\section{Conclusión}

Con el objetivo propuesto de identificar los esquemas urbanos apropiados para disminuir las temperaturas estivales y la consecuente demanda de energía para el acondicionamiento térmico de los espacios residenciales, fue necesaria una caracterización que permitiera ordenar y diferenciar los variados descriptores que intervienen en su diseño.

De esta caracterización se pudo observar cómo las variaciones que poseen las tramas analizadas modifican sus comportamientos térmicos. Esto implica que tramas ubicadas en sectores cercanos y que poseen la misma orientación, presentan diferencias térmicas que varían de acuerdo con un conjunto de indicadores o variables, y son el resultado de sus combinaciones con las características del clima local. En este sentido, un análisis estadístico de estas interrelaciones ha permitido establecer, en los resultados, criterios mínimos de diseño que posibilitarían mejorar el desempeño térmico y energético de las áreas residenciales localizadas en el piedemonte de la ciudad de Mendoza, Argentina.

Mediante el análisis de componentes principales, se pudo explicar el grado de dependencia de las temperaturas urbanas y detectar, de un conjunto de 19 variables o indicadores seleccionados inicialmente para caracterizar los espacios evaluados, aquellos de mayor peso. Con esto fue posible reducir a seis las variables que explican o permitirían controlar el comportamiento de la temperatura urbana a lo largo del día: azimut, factor de visión de cielo (SVF), relación entre patios centrales y perimetrales, densidad, factor de ocupación de superficie (FOS) y horas de sol (HS).

Si bien el análisis estadístico brinda un diagnóstico y una mejor comprensión de cómo interactúan el clima y las variables de diseño urbano, en futuros trabajos se propone realizar simulaciones y detectar los rangos en los cuales las combinaciones de indicadores propuestos ofrecen las mejores posibilidades de diseño a lo largo de todo el día. A partir de ello, se pueden proponer alternativas para climatizar las ciudades a través de un diseño urbano que influya positivamente sobre el comportamiento térmico de la región de piedemonte, con el objetivo de mitigar el desarrollo de la isla de calor, mejorar la habitabilidad de los espacios públicos y disminuir los consumos de energía eléctrica de los edificios.

Por último, cabe destacar que la planificación del crecimiento urbano da como resultado alternativas sustentables para el desarrollo de las áreas de piedemonte en toda su complejidad. En estas últimas décadas se propusieron esquemas urbanos que continuaron estructuras presentes en la llanura del AMM, caracterizados por: 
- una trama urbana del tipo racional, con manzanas rectangulares con orientación N-S, E-O, distribuida porcentualmente de manera equitativa (30\%) (Sosa, 2018);

- una configuración espacial del tipo abierto -es decir, baja compacidad-, resultante de canales viales anchos de 20 o $30 \mathrm{~m}$ y baja densidad edilicia, determinada por construcciones de un nivel $(3 \mathrm{~m})$;

- diseño heterogéneo de la forestación tanto en lo que respecta a diversidad de especies por cuadra como a intervalos de plantación;

- alto sellamiento de suelo con valores fos iniciales de 35\% que, luego de ampliadas las viviendas, resultan superiores a 60\%; y

- concentración de área libre en el centro de la manzana.

Sin embargo, este trabajo demuestra que para mejorar el desempeño térmico de verano de los espacios públicos en áreas residenciales localizadas en el piedemonte, debe propiciarse la orientación de la trama N-S y SE-NO (menores valores de azimut); tender a configuraciones espaciales compactas; seleccionar y distribuir las especies forestales para que resulten apropiadas al perfil del canal vial y permitan una adecuada protección durante el día, sin interferir con las posibilidades de enfriamiento radiativo nocturno; disminuir los valores de Fos de modo de reducir el sellamiento de suelos; incrementar la proporción de áreas libres en relación al área sellada; y tender a la distribución de las áreas libres en el contexto de la manzana.

Finalmente, de lo descrito se desprende que los modelos de urbanización no son replicables en geomorfologías diferentes y que la eficiencia de un modelo de desarrollo urbano está asociado al aprovechamiento de los recursos naturales y al equilibrio entre los esquemas de diseño y las variables climáticas, topográficas y territoriales.

\section{Referencias bibliográficas}

Abraham, M., Roig, F. \& Salomón, M. (1990). Proyecto: Planificación y ordenamiento del piedemonte al oeste de la ciudad de Mendoza. Mendoza: Instituto Argentino de Investigaciones de Zonas Aridas (Iadiza). http://www.asicprimerazona.com.ar/asic/ publicaciones/planif_oeste_mza.pdf

Balmaceda, M., Cantón, M. \& Correa, E. (2018). Impacto microclimático estival de la configuración de distintas tipologías de espacio abierto en manzanas urbanas. Esquema concentrado versus disperso. EURE, 44(133), 233-253. http://doi.org/10.4067/ s0250-71612018000300233

Castillo, A., Correa, E. \& Cantón, M. (2016). Desarrollo urbano sostenible de áreas de piedemonte. El caso del Área Metropolitana de Mendoza. I Encuentro Nacional sobre Ciudad, Arquitectura y Construcción Sustentable (ENACS 2016) (pp. 555-566). Facultad de Arquitectura y Urbanismo. La Plata, Argentina. http://hdl.handle.net/10915/59476 
Castillo, A., Correa, E. \& Cantón, M. (2017). Regulación urbanística de áreas de piedemonte en ciudades latinoamericanas. Análisis crítico de la normativa internacional y el marco regulatorio de la ciudad de Mendoza, Argentina. [en línea]. ACE: Architecture, City and Environment - Arquitectura, Ciudad y Entorno, 12(35), 39-70. http://doi.org/10.5821/ ace.12.35.4761

Correa, E., De Rosa, C. \& Lesino, G. (2006). Isla de calor urbana. Distribución espaciotemporal de temperaturas dentro del área metropolitana de Mendoza. Avances en Energias Renovables y Medio Ambiente, 10(11), 121-128. https://www.mendozaconicet.gob.ar/asades/modulos/averma/trabajos/2006/2006-t011-a018.pdf

Correa, E., Pattini, A. \& Fornés, M. (2006). Software: Pixel de Cielo. Programa informático que calcula el factor de visión de cielo en un punto determinado de un área de estudio, a partir del procesamiento de una imagen digital hemisférica tomada con una lente ojo de pez. Número de registro CONICET: 07304 (2006-VIGENTE).

De la Fuente Fernández, S. (2011). Componentes principales - ACP. Madrid: Universidad Autónoma de Madrid (UAM), Facultad de Ciencias Económicas y Empresariales. http:// www.fuenterrebollo.com/Economicas/ECONOMETRIA/MULTIVARIANTE/ ACP/ACP.pdf

Di Rienzo, J., Casanoves, F., Balzarini, M., González, L. Tablada, M. \& Robledo, W. (2011). InfoStat Versión 2011. Grupo InfoStat. Universidad Nacional de Córdoba, Argentina, Facultad de Ciencias Agropecuarias (FCA).

Filippín, C., Ricard F. \& Flores Larsen, S. (2013). Evaluation of heating energy consumption patterns in the residential building sector using stepwise selection and multivariate analysis. Energy and Buildings, (66), 571-581. https://doi.org/10.1016/j. enbuild.2013.07.054

Givoni, B. (1991). Comfort, climate analysis and building design guidelines. Energy and Building, 18(1), 11-23. https://doi.org/10.1016/0378-7788(92)90047-K\}

Instituto Nacional de Estadísticas y Censos (Indec), República Argentina (2010). Censo Nacional de Población, Hogares y viviendas 2010 [en línea]. http://www.censo2010. indec.gov.ar/

Instituto Provincial de la Vivienda (IPv), Mendoza [en línea]. http://www.ipvmendoza.gov.ar/

Libertun de Duren, N. (2014). Resumen Ejecutivo: Cumbre de América Latina y China sobre Políticas de Desarrollo Urbano y Vivienda. En Cumbre de América Latina y China de Politicas y Conocimiento: Impactos de la urbanización rápida y la prosperidad nacional (pp. 9-11), realizada en Lima, Perú, 15 y 16 de julio de 2014, y coorganizada por la Academia China de Ciencias Sociales, el Ministerio de Vivienda, Construcción y Saneamiento de Perú y el Banco Interamericano de Desarrollo. Nueva York: Banco Interamericano de Desarrollo. https://publications.iadb.org/handle/11319/6732

López, J. \& López, C. (2004). El urbanismo de ladera: un reto ambiental, tecnológico y del ordenamiento territorial. Bitácora Urbano Territorial, 1(8), 94-102. http://www. redalyc.org/articulo.oa?id=74800814

Oke, T. (2004). Initial guidance to obtain representative meteorological observations at urban sites. IOM Report. Geneva: World Meteorological Organization, 81(1250), 51. http:// blogs.ubc.ca/toke/files/2015/12/IOM-81-UrbanMetObs.pdf 
Romero, H. \& Vásquez, A. (2005). Evaluación ambiental del proceso de urbanización de las cuencas del piedemonte andino de Santiago de Chile. EURE, 31(94), 97-117. https:// doi.org/10.4067/S0250-71612005009400006

Ruiz, M., Sosa M., Correa, E. \& Cantón, M. (2015). Suitable configurations for forested urban canyons to mitigate the UHI in the city of Mendoza, Argentina. Urban Climate, 14(2), 197-212. https://doi.org/10.1016/j.uclim.2015.05.005.

Secretaría de Ambiente y Ordenamiento Territorial (2017). Sistema de Información Ambiental Territorial (SIAT). Crecimiento urbano hacia el piedemonte, por períodos. [en línea] http://www.siat.mendoza.gov.ar/data/geonode:crecimiento_urbano

Welz, J. \& Krellenberg, K. (2016). Vulnerabilidad frente al cambio climático en la Región Metropolitana de Santiago de Chile: posiciones teóricas versus evidencias empíricas. EURE, 42(125), 215-272. https://doi.org/10.4067/S0250-71612016000100011 